\title{
A photometric-dynamic model to simulate coma and jets from a comet
}

\author{
Application to comet Hale-Bopp (C/1995 01)
}

\author{
R. Vasundhara ${ }^{\star}$ \\ Indian Institute of Astrophysics, Bangalore 560034, India
}

Received 29 December 1998 / Accepted 5 November 2001

\begin{abstract}
A model to compute the trajectories of dust grains ejected by comets to simulate intensity and polarization maps on the sky plane is presented. The model is used to constrain the silicate to organic mass ratio, porosity and size distribution of the grains in the shells and coma of comet Hale-Bopp to explain 1) the published polarizations at three continuum wavelengths at $0.6840 \mu \mathrm{m}, 0.4845 \mu \mathrm{m}$ and $0.3650 \mu \mathrm{m}$ at different phase angles, 2) higher polarization and bluer colour of the shells compared to the coma, 3) the intensity variation and rapid decline of polarization on the sunward side and 4) higher polarization on the anti-sunward direction. The present results obtained using Mie theory in the model indicate that although it is impossible to determine the nature of the silicate (pyroxenes/olivines or amorphous/crystalline) using the present technique, the porosity, organic fraction and size distribution of the grains can be constrained. The fit to the data set indicates an ensemble of grains in which $\approx 60 \%$ are silicate grains with a range in silicate to organic mass ratio $r_{\mathrm{m}}$ between 1 and 2 and of medium porosity $<50 \%$. A higher silicate content of $r_{\mathrm{m}}>8$ on the shells can explain their bluer colour. A narrow size distribution between $0.08 \mu \mathrm{m}-0.6 \mu \mathrm{m}$ is inferred for $70 \%$ of the grains in the coma and shell. These small grains may not be individual grains but part of loosely bound grains in which larger grains may also be present which may control the dynamics. The narrow size distribution may therefore represent statistically the size range of individual subunits of larger highly porous aggregates of fractal dimension close to two. The polarizations in $J H K$ bands also support the aggregate structure. Despite the availability of an extensive and wide range of data set on this comet, the inclusion of a large parameter space, lack of knowledge on the nature of organic material found in comets and use of Mie theory, which does not adequately represent the polarization phase curves of natural grains, set limitations in getting a unique solution in the present work. The results presented here are open to improvements when more realistic light scattering theories are used in the model and our understanding on the nature of the organic content of the grains improves. However, visibility of up to 8-10 shells in well exposed images implies $\beta<1$ for these grains. As a result of this dynamical constraint which is independent of the assumptions on the scattering characteristics of the grains, the results for the dust grains in the shell may be more robust compared to that of the coma.
\end{abstract}

Key words. comets - Hale-Bopp: scattering - polarization - colour

\section{Introduction}

Compositions of the silicate grains in the coma of comet Hale-Bopp have been derived from the spectral emission signatures in the $10 \mu \mathrm{m}$ region by Crovisier et al. (1997), Hayward \& Hanner (1997) and Wooden et al. (1999). Hayward et al. (2000) from their thermal infrared imaging and spectroscopy of Comet Hale-Bopp found that the silicate features were up to $15 \%$ stronger in the jets and halos compared to regions between the halos. Further, the

* e-mail: rvas@iiap.ernet.in leading edge of the shells were found by these authors to have higher color temperature and silicate/continuum flux ratio. Variation of the silicate features from central coma to the first two shells has been reported by Galdemard et al. (1997).

In the optical and near IR regions, extensive polarization observations at a range of solar phase angles have been reported by Ganesh et al. (1998), Hadamcik et al. (1997), Hasegawa et al. (1999), Jockers et al. (1997), Jones \& Gehrz (2000), Manset \& Bastien (1998) and Tanga et al. (1997). Use of the technique of imaging polarimetry by 
most of these groups yielded valuable information on the spatial variation of polarization. Polarization was found to be high in the anti-sunward direction and in the new shells but rapidly declined in the older shells in the sunward direction. Information on the colour of the grains has been reported by Kiselev \& Velichko (1997), Kolokolova et al. (2001) and Bellucci (1998). The full potential of these spatially resolved infrared, colour and polarimetric observational data can be harvested to investigate the porosity $p$, silicate to organic mass ratio $r_{\mathrm{m}}=m_{\mathrm{sil}} / m_{\mathrm{org}}$ of the grains and their size distributions separately in the coma and the shells. The present work describes a photometric model to map the trajectory of the grains and to compute the theoretical brightness distribution, colour and polarization in the coma and the shells for comparison with these reported observations.

\section{The model}

The computer simulations are based on the basic concepts introduced by Sekanina $(1981,1991)$ and Sekanina \& Larson (1984). Except for the outburst events during 1995, which were sporadic activities, the dust grains are assumed to be ejected continuously from the sources radially outwards, from local sunrise to sunset. The ejected grains are subjected to the gravitational attraction of the Sun and the Solar radiation pressure force in the opposite direction. In the present work, since we study the shells which are close to the nucleus within a distance of $\approx 100000 \mathrm{~km}$, it is assumed that dust grains follow the same Keplerian motion as that of the comet due to solar gravity. Thus, relative to the comet, the position of the dust grain can be calculated from its initial velocity, the radiation pressure force and the ejection geometry.

\subsection{Velocity and acceleration of the grains}

The velocity $v_{\text {gr }}$ attained by the grains due to gas drag (Probstein 1969) can be calculated using the relation by Sekanina (1981):

$1 / v_{\mathrm{gr}}=a+b / \sqrt{\beta}$

and also the following equation by Fulle (1987)

$v_{\mathrm{gr}}=v_{0} \beta^{1 / 6}$

where $\beta$ is the ratio of the force due to solar radiation pressure on the grain to the gravitational force. Sekanina \& Larson (1984) have used Eq. (1) successfully for emission from discrete sources, and pointed out that it is valid for slightly absorbing grains with $\beta \leq 0.6$. The acceleration $\alpha$ due to solar radiation pressure depends on the nature of the grain and the heliocentric distance. Using the definition of $\beta$,

$\alpha=\left(\beta g_{\operatorname{sun}(1)}\right) / r^{2}$,

where $g_{\text {sun(1) }}$ is the acceleration due to solar gravity at one $\mathrm{AU}\left(0.6 \times 10^{-5} \mathrm{~km} \mathrm{~s}^{-2}\right)$ and $r$ the heliocentric distance of the comet. The value of $\beta$ can be calculated for a grain of given size and composition using the relation by Finson \& Probstein (1968):

$$
\begin{aligned}
\beta & =F_{\mathrm{rad}} / F_{\mathrm{grav}} \\
& =\frac{3 Q_{\mathrm{pr}} E_{\odot}}{4 \pi c G M_{\odot}} \frac{1}{\rho s}
\end{aligned}
$$

where $Q_{\mathrm{pr}}$ is the scattering efficiency for radiation pressure of the grain, $s$ its radius and $\rho$ its density. $E_{\odot}$ is the total solar radiation per second, $c$ the velocity of light, $M_{\odot}$ the mass of the Sun and $G$ the universal gravitational constant. In the present investigation, the grain size is allowed to vary from $s_{\min }=0.05 \mu \mathrm{m}$ to $s_{\max }=30.0 \mu \mathrm{m}$ with Hanner (1985) size distribution law. Since the radiation pressure parameter $Q_{\mathrm{pr}}$ of a grain material depends both on its size and the wavelength of radiation, the value of $\beta$ for a grain of radius $s$ was calculated using the more precise relation

$\beta=\frac{3}{4 \pi c G M_{\odot}} \frac{1}{\rho s} \int_{\lambda 1}^{\lambda 2} Q_{\mathrm{pr}}(s, \lambda) F_{\odot}(\lambda) \mathrm{d} \lambda$,

where, $F_{\odot}(\lambda)$ is the mean total solar radiation from the sun per second per Angstrom at the wavelength $\lambda$. The above integration was carried out from $\lambda 1=0.1 \mu \mathrm{m}$ to $\lambda 2=5.0 \mu \mathrm{m}$.

\subsection{Comet-o-centric spherical coordinates of the grains referred to comet's equator}

In the simulation, the path of the dust grain ejected radially from an active region at a comet-o-centric longitude $u$, latitude $\phi$ and the nuclear radius $R$ is calculated as a function of time. Figure 1 shows the ideal case of a spherically symmetric nucleus centered at C. The sub-Sun and sub-Earth points are $S$ and $E$ respectively and $N_{\mathrm{C}}$ is the North pole of the comet. The direction of the Earth's north pole is along $C P_{\mathrm{E}}$. The ascending node of the comet's equator on the Earth's equator is $N_{\text {equ }}$ and that on the ecliptic is indicated by $N_{\text {ecl }}$. Components of $\alpha$ parallel to the plane of the comet's equator and perpendicular to it are $\alpha_{\|}=\alpha \cos B^{\prime}$ parallel to $S F$ and $\alpha_{\perp}=\alpha \sin B^{\prime}$ parallel to $S T$ respectively where $B^{\prime}$ is the comet-o-centric latitude of the Sun. The longitude of the source and the dust grains are measured from $N_{\text {equ }}$ along the direction of rotation of the comet. The longitude of the sub-Earth point $E$ is $U-\pi$ and the angle $B=\widehat{E_{\mathrm{E}} C E}$ is its latitude. Dust grains are ejected radially outwards with the velocity $v_{\text {gr }}$ from an active source $G(u, \phi, R)$, where $u=N_{\text {equ }} E_{\mathrm{D}}$ and $\phi=E_{\mathrm{D}} G$. Strictly speaking, the grain attains this velocity after it is dragged by the subliming gas to a few nuclear radii. However, considering the vast spatial extent of the shell and jet structures of several rotations which are being modeled, this distance can be ignored. Relative to the comet, during time $t$, the dust traverses a distance $v_{\mathrm{gr}} t$ in the radial direction and a distance $1 / 2 \alpha t^{2}$ along the Sun - comet direction due to solar radiation pressure. 


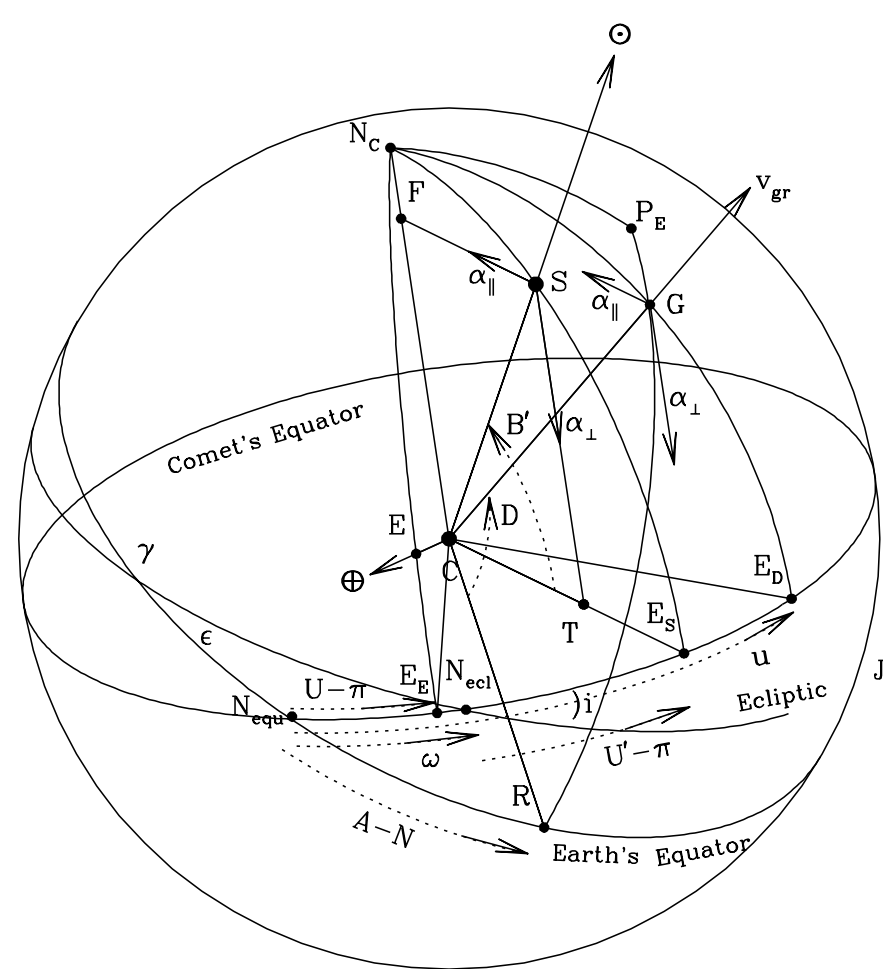

Fig. 1. A model to compute the sky plane coordinates of the ejected dust grains. The comet centered at $\mathrm{C}$ is assumed to be spherically symmetric. The grain from the source at $G$ is ejected with velocity $v_{\mathrm{gr}}$. The north pole of the comet is at $N_{\mathrm{C}}$, $C P_{E}$ is the direction of Earth's celestial pole, $E$ and $S$ are the sub-Earth and sub-Sun points respectively. See text for more details.

The position $\left(u^{\prime}, \phi^{\prime}, r^{\prime}, t\right)$ of the grain at time $t$ reckoned after ejection is given by:

$r^{\prime} \cos \phi^{\prime} \cos u^{\prime}=v_{\mathrm{gr}} t \cos \phi \cos u-(1 / 2) \alpha t^{2} \cos B^{\prime} \cos U^{\prime \prime}$

$r^{\prime} \cos \phi^{\prime} \sin u^{\prime}=v_{\mathrm{gr}} t \cos \phi \sin u-(1 / 2) \alpha t^{2} \cos B^{\prime} \sin U^{\prime \prime}$

$r^{\prime} \sin \phi^{\prime}=v_{\mathrm{gr}} t \sin \phi-(1 / 2) \alpha t^{2} \sin B^{\prime}$

where $U^{\prime \prime}$, the longitude of the sub-Sun point is given by

$U^{\prime \prime}=N_{\text {equ }} E_{\mathrm{S}}=\omega+U^{\prime}-\pi$.

The expressions in Eq. (7) are valid provided the direction and magnitude of $\alpha$ is constant. For the observations during February-May 1997, since we model only up to a maximum of 8 shells, a constant value for $\alpha$ during the period of 8 rotations may be a valid assumption for comet HaleBopp with a period of $11.34 \mathrm{hr}$ (Jorda et al. 1997; Licandro et al. 1998). For the 1995-96 images, slow apparent motion of the comet again justifies such an assumption.

The angles $\omega=N_{\text {equ }} N_{\text {ecl }}, U^{\prime}-\pi=N_{\text {ecl }} E_{\mathrm{S}}$ can be calculated from the following relations:

$$
\begin{aligned}
& \sin \omega \sin i=\sin \epsilon \sin N \\
& \cos \omega \sin i=\cos \epsilon \sin J-\sin \epsilon \cos J \cos N \\
& \cos i=\cos \epsilon \cos J+\sin \epsilon \sin J \cos N,
\end{aligned}
$$

where $i$ is the inclination of the comet's equator to the ecliptic. The angles $N=\gamma N_{\text {equ }}$ and $J$, the inclination of the comet's equator to the Earth's equator are related to the right ascension and declination of the comet's pole:

$N=\alpha_{p}+\pi / 2$

$J=\pi / 2-\delta_{\mathrm{p}}$.

The angles $U, U^{\prime}, B, B^{\prime}$ and the position angle $P$ of the north pole of the comet projected on the sky plane were calculated using the equations used for calculating the planet-o-centric positions of the satellite with respect to the planets (Rhode \& Sinclair 1992):

$$
\begin{aligned}
& \cos B \sin U=\cos J \cos \delta \sin (\alpha-N)+\sin J \sin \delta \\
& \cos B \cos U=\cos \delta \cos (\alpha-N) \\
& \sin B=\sin J \cos \delta \sin (\alpha-N)-\cos J \sin \delta \\
& \cos B \sin P=-\sin J \cos (\alpha-N) \\
& \cos B \cos P=\sin J \sin \delta \sin (\alpha-N)+\cos J \cos \delta \\
& \cos B^{\prime} \sin U^{\prime}=\cos i \cos b \sin (l-\Omega)+\sin i \sin b \\
& \cos B^{\prime} \cos U^{\prime}=\cos b \cos (l-\Omega) \\
& \sin B^{\prime}=\sin i \cos b \sin (l-\Omega)-\cos i \sin b,
\end{aligned}
$$

where $\alpha, \delta, l$ and $b$ are the geocentric right ascension, declination, heliocentric ecliptic longitude and latitude of the comet respectively. The angle $\Omega=\gamma N_{\text {ecl }}$ can be calculated using the following relations:

$$
\begin{aligned}
\cos \Omega \sin i & =-\cos J \sin \epsilon+\sin J \cos \epsilon \cos N \\
\sin \Omega \sin i & =\sin N \sin J
\end{aligned}
$$

The angles $U, P$ and $B$ are not directly needed for calculating the trajectory of the dust grain. These are nevertheless useful to visualize the defect of solar illumination on the comet, the orientation and aspect angle of the dust jet and shell patterns.

\subsection{Comet-o-centric spherical coordinates of the dust grain referred to the Earth's equator}

The comet-o-centric coordinates $\left(u^{\prime}, \phi^{\prime}, r^{\prime}, t\right)$ referred to the comet's equator were calculated using Eq. (7). These were transformed to the comet-o-centric Earth's equatorial coordinates $\left(A^{\prime}, D^{\prime}, r^{\prime}, t\right)$ of the grain using the following equations:

$\cos \left(A^{\prime}-N\right) \cos D^{\prime}=\cos \phi^{\prime} \cos u^{\prime}$ $\sin \left(A^{\prime}-N\right) \cos D^{\prime}=\cos \phi^{\prime} \sin u^{\prime} \cos J-\sin \phi^{\prime} \sin J$ $\sin D^{\prime}=\cos \phi^{\prime} \sin u^{\prime} \sin J+\sin \phi^{\prime} \cos J$.

The comet-o-centric equatorial coordinate of the source at $G(A, D, R, t=0)$ is shown in Fig. 1 .

\subsection{Differential sky plane coordinates of the dust grain with respect to the comet's center}

The projected location of the grain on the sky plane with respect to the comet center is obtained by computing its 
differential Earth's equatorial co-ordinates using the following equations (Gurnette \& Woolley 1960):

$\tan \left(\alpha_{\mathrm{D}}-\alpha\right)=\frac{\xi}{(1+\zeta) \cos \delta-\eta \sin \delta}$

$\tan \left(\delta_{\mathrm{D}}-\delta\right)=\frac{\eta-\xi \tan \frac{1}{2}\left(\alpha_{\mathrm{D}}-\alpha\right) \sin \delta}{(1+\zeta)+\xi \tan \frac{1}{2}\left(\alpha_{\mathrm{D}}-\alpha\right) \cos \delta}$

where

$\xi=r^{\prime} \cos D^{\prime} \sin \left(A^{\prime}-\alpha\right) / \Delta$

$\eta=r^{\prime}\left(\sin D^{\prime} \cos \delta-\cos D^{\prime} \sin \delta \cos \left(A^{\prime}-\alpha\right)\right) / \Delta$

$\zeta=r^{\prime}\left(\sin D^{\prime} \sin \delta+\cos D^{\prime} \cos \delta \cos \left(A^{\prime}-\alpha\right)\right) / \Delta$,

where $\alpha_{\mathrm{D}}$ and $\delta_{\mathrm{D}}$ are the geocentric right ascension and declination of the grain and $\Delta$ its geocentric distance. These rigorous expressions in Eqs. (13)-(17) are actually used for calculating the differential coordinates of satellites with respect to the primary without any assumptions regarding their latitudes. These are therefore applicable in the present case of the comet-dust geometry.

\section{Brightness estimates of the shells and the coma}

The intensity of light $I_{\mathrm{S}}(\theta, \lambda)$ and polarization $P_{\mathrm{S}}(\theta, \lambda)$ scattered by the grain towards the observer are given by (Krishnaswamy \& Shah 1987):

$$
\begin{aligned}
I_{\mathrm{S}}(\theta, \lambda) & =\frac{I_{0}(\lambda) \lambda^{2}}{8 \pi r^{2} \Delta^{2}}\left(i_{\perp}+i_{\|}\right) \\
P_{\mathrm{S}}(\theta, \lambda) & =\left(i_{\perp}-i_{\|}\right) /\left(i_{\perp}+i_{\|}\right)
\end{aligned}
$$

where $\theta$ is the scattering angle and $I_{0}(\lambda)$ is the solar intensity at $1 \mathrm{AU}$. The intensity scattering functions $i_{\perp} \&$ $i_{\|}$polarized perpendicular and parallel to the scattering plane, and the scattering efficiency for radiation pressure $Q_{\mathrm{pr}}(s, \lambda)$ of the grain of radius $s$ at the wavelength of radiation $\lambda$ (Eq. (6)) were estimated using Mie scattering using the code BHMIE by Bohren \& Huffman (1983).

The position of the grain in the shell projected on the sky plane at a given time was thus calculated using Eqs. (13) and (14) and the intensity and polarization of light scattered by it was computed using the Eqs. (18) and (19). The colour is calculated following the definition by Jewitt \& Meech (1986) as the normalized reflectivity gradient expressed in $\%, S^{\prime}\left(\lambda_{1}, \lambda_{2}\right)=(\mathrm{d} S / \mathrm{d} \lambda) \times$ $1000 / S_{\text {mean }}$ between wavelengths $\lambda_{1}$ and $\lambda_{2}$, where $\mathrm{d} S / \mathrm{d} \lambda$ is the rate of change of reflectivity with wavelength and $S_{\text {mean }}$ is the mean reflectivity.

\section{Application of the model}

\subsection{Observational inputs}

1. Image of comet Hale-Bopp on 10 April, 1997 in $R$ filter obtained from the Vainu Bappu observatory was used to investigate the intensity variations in the shells and the coma.
2. Published polarizations on 10 June, 1996, 30 September, 1996, and 9th April, 1997 separately in the coma and bright regions by Hadamcik et al. (1997) were used to investigate the nature of the grains in the coma.

3. As the colour of the grains do not change drastically with phase angle, we set a conservative limit of $5-10 \%$ per $1000 \AA$ on the shells and $10-20 \%$ per $1000 \AA$ on the regions between the shells representing the coma based on the colour reported by Bellucci (1998), Furusho et al. (1999), Kiselev \& Velichko (1997) and Kolokolova et al. (2001).

4. Published aperture polarimetric observations at a range of phase angles by several groups (Sect. 1 and references therein).

\subsection{Selection of grain material}

Cometary grains are known to be porous and contain an organic component (Greenberg \& Hage 1990; Hage \& Greenberg 1990; Krishnaswamy et al. 1988; Xing \& Hanner 1997). The dynamics as well as the scattering properties of the grains are affected by porosity and organic content, as both these factors modify the complex refractive indices of the grains significantly. The silicaceous components in the grain have been identified from their emission signatures in the IR spectra in the $10 \mu \mathrm{m}$ region by Hayward et al. (2000) and Wooden et al. (1999). In the visible and near IR regions, it is futile to identify the $\mathrm{Mg}$ rich silicates either in glassy or crystalline form in the composite grains using their scattered radiation, as presence of even a small amount of organic content of $1 / 12$ by weight will be sufficient to significantly alter the refractive index of the composite grain. Hence silicates were sub grouped into three classes depending on the absorption index $k$ :

1. $\mathrm{Mg}$ rich silicates with $0.0001<k<0.001$, we refer to this group as SiX. Forsterite, with the optical constants by Scott \& Duley (1996) was taken as the sample representative of this class (class 1 ).

2. Astronomical silicate (Draine 1985; Draine \& Lee 1984) with $k \approx 0.03$ (SiA). The present study uses the constants of crystalline olivine constructed by $\mathrm{Li}$ \& Greenberg (1997) which uses the absorptive index of $\mathrm{SiA}$ in the visible region. Since polarization mainly depends on the absorptive index, we refer to this sample also as $\mathrm{SiA}$ (class 2).

3. $\mathrm{Mg}$ poor silicates characterized by an absorption at wavelengths shortward of $0.5 \mu \mathrm{m}$ and with $k>0.03$. Amorphous olivine (Aol) and pyroxene (Apy) with $\mathrm{Mg}$ number of 0.5 (Dorschner et al. 1995) were taken as representatives of this class (class 3).

At $r_{\mathrm{m}} \leq 12$, distinction between SiA and SiX almost vanishes. Following Li \& Greenberg (1998), the organic refractory component was assumed to be a composite of interstellar refractory (Li \& Greenberg 1997) and amorphous carbon (Rouleau \& Martin 1991) of equal mass. Effective refractive indices of the composite grains were determined 
Table 1. Size distributions investigated in the present study.

\begin{tabular}{llllcc}
\hline Distribution & $s_{\mathrm{o}}$ & $N^{\prime}$ & $M^{\prime}$ & \multicolumn{2}{c}{ Grain sizes } \\
& $\mu \mathrm{m}$ & & & $\begin{array}{c}s_{\min } \\
\mu \mathrm{m}\end{array}$ & $\begin{array}{c}s_{\max } \\
\mu \mathrm{m}\end{array}$ \\
\hline$a^{1}$ & 0.001 & 3.85 & 92 & 0.05 & 30 \\
$b^{2}$ & 0.05 & 3.70 & 8.47 & 0.05 & 30 \\
$c^{1}$ & 0.1 & 3.60 & 12.9 & 0.10 & 30 \\
\hline
\end{tabular}

1. Derived in the present work.

2. Adopted from Hayward et al. (2000).

using Bruggmann's mixing rule. Collections of individual grains of these silicates with the organic refractory component in the mass ratios $r_{\mathrm{m}}=0,1,2,8,12$ and $\infty$ and the porosities $p=0 \%, 30 \%, 35 \%, 40 \%, 45 \%, 50 \%, 60 \%$, $70 \%, 80 \%$ and $95 \%$ were considered.

\subsection{Short-listing of grain types - preliminary fit to three colour phase vs. polarization data}

Phase dependence of polarization carries valuable information on the interaction between the reflected and refracted components from the grain along different scattering directions. The fraction of the refracted component depends on the optical properties of the grain, its size and shape. Polarization of light scattered by an ensemble of spherical grains at the three continuum wavelengths $0.6840 \mu \mathrm{m}, 0.4845 \mu \mathrm{m}$ and $0.3650 \mu \mathrm{m}$ corresponding to aperture polarimetry observations by Ganesh et al. (1998) and Manset \& Bastien (1998) were computed. For a proper match with these observations over the entire range of phase angles, combinations of grain types and proper choice of their size distribution are essential. Hanner size distribution (Hanner 1985)

$n(s) \mathrm{d} s=\left(1-s_{0} / s\right)^{M^{\prime}}\left(s_{0} / s\right)^{N^{\prime}}$

was used. The constants $s_{0}, M^{\prime}$ and $N^{\prime}$ determine the slopes at the lower and upper size domains and the radius $s_{\mathrm{p}}$ at which $n(s)$ is maximum. As a first guess, four component equal weight inter-combinations of the three classes of silicates, each with 30 different combinations of $p$ and $r_{\mathrm{m}}$ were considered. A total of 810000 combinations were compared in the least square sense with the observed phase curves of Ganesh et al. (1998). The size distribution parameters were varied to improve the $\chi^{2}$ values. The constants in set ' $a$ ' in Table 1 yielded best fit. The intensity contribution $I(s) n(s) \mathrm{d} s$ for this distribution at a mean wavelength of $0.5 \mu \mathrm{m}$ is shown as the solid line in Fig. 2. Table 2 gives the grain types which were common in the first 100 best fitting combinations. It is striking to note that except for the highly porous grains, all grain types have porosities in the range between $0 \%$ (compact) and $50 \%$.

The size distribution ' $a$ ' represents the combined grain population in the coma and shell which are sampled in the aperture of $26^{\prime \prime} 5$. In the present work it is attempted to

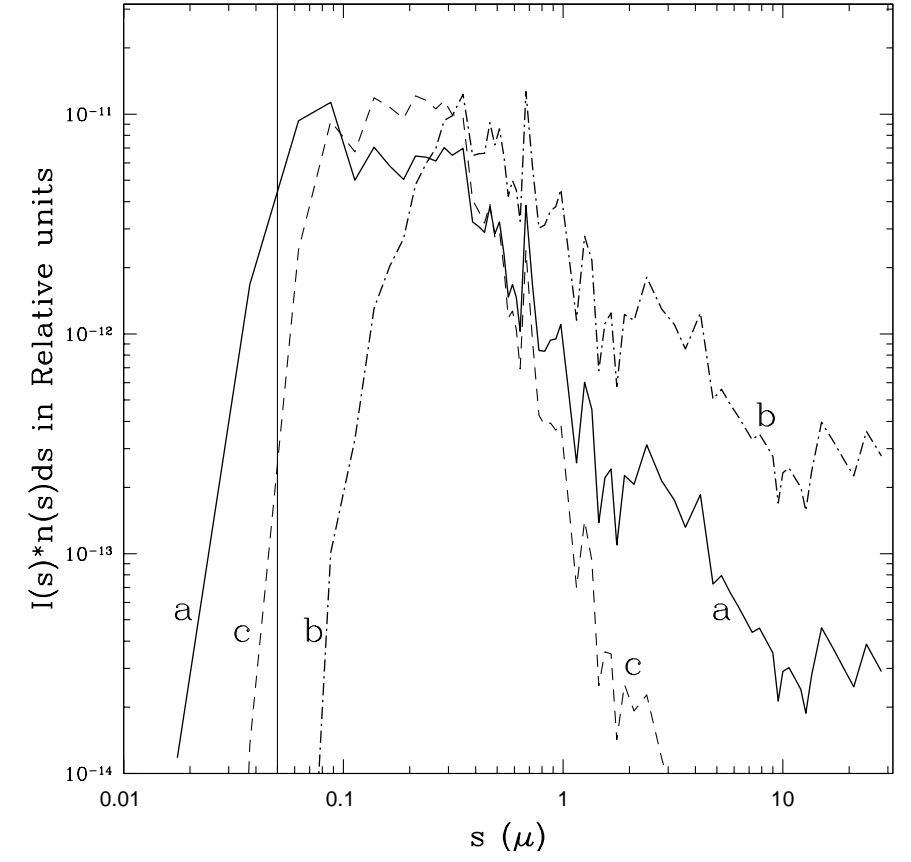

Fig. 2. Intensity contribution of grains of sizes between $s$ and $s+\mathrm{d} s$, along the scattering angle of $136^{\circ}$ and at a wavelength of $0.5 \mu \mathrm{m}$ for the size distribution constants in Table 1 . All the three curves have been normalized to enable a direct comparison. The solid vertical line indicates the lower limit of $0.05 \mu \mathrm{m}$ on the grain size used in the present investigation.

Table 2. Short-listed grain types.

\begin{tabular}{llllll}
\hline & $\begin{array}{c}\mathrm{Mg} \text { rich } \\
\mathrm{SiA}^{1} /\end{array}$ & $\mathrm{SiA}$ & $\mathrm{SiX}$ & $\begin{array}{l}\mathrm{Mg} \text { poor }_{\mathrm{Aol}^{3} /} \\
\mathrm{Apy}^{4}\end{array}$ & $\begin{array}{l}\mathrm{SiA} / \mathrm{SiX} / \\
\mathrm{Aol} / \mathrm{Apy}^{5}\end{array}$ \\
\hline$r_{\mathrm{m}}$ & $1,2,8,12$ & $\infty$ & $\infty$ & $\infty$ & $1-\infty$ \\
$p(\%)$ & $0-50$ & $30-50$ & $30-50$ & $30-50$ & 95 \\
\hline
\end{tabular}

1. Close to Astronomical silicate.

2. Olivine or Pyroxene in crystalline or amorphous state.

3. $\mathrm{Mg}_{2 y} \mathrm{Fe}_{2-2 y} \mathrm{SiO}_{4}$ with $y=.5$.

4. $\mathrm{Mg}_{x} \mathrm{Fe}_{1-x} \mathrm{SiO}_{3}$ with $x=.5$.

5. At $p \geq 90 \%, r_{\mathrm{m}}$ is poorly constrained. These grains may represent porous grains with Rayleigh type inclusions.

investigate the relative fraction of the grain type and their size distributions separately in the coma and the shells.

\subsection{Dust emission geometry}

The simulations of the intensity, colour and polarization maps of comet Hale-Bopp were carried out for the geometry on 10 April, 1997, in the wavelength band corresponding to the cometary continuum wavelengths at $0.4450 \mu \mathrm{m}$ and $0.6840 \mu \mathrm{m}$ for the grain types in Table 2 . The pole positions and latitude of the sources are adapted from Paper II. The grain velocities computed using Eq. (2) with $v_{0}=0.63 \mathrm{~km} \mathrm{~s}^{-1}$ were found to be within $\pm 5 \%- \pm 10 \%$ to the values computed using Eq. (1). The latter was used in the simulations. The coma was simulated by considering 
emission from a grid on the comet at intervals of $10^{\circ}$ in latitude and $10^{\circ} \cos (\phi)$ in longitude, where $\phi$ is the latitude of the point on the grid. The sources are assumed to be active when sunlit. The trajectories of the dust grains are computed assuming collimated emissions. In reality, the emission may be spread over a cone. Further, the velocity of the grains will have a spread due to deviation from their assumed sphericity, finite size of the active regions and possible irregular local terrain. Their cumulative effect will produce a broadening of the shells. As it is difficult to model each of these effects, the sky plane position of the grains were convolved with a Gaussian-random profile with a sigma of 1 ".5. To include the effect of spreading with time, the sigma was allowed to increase by 0.25 per day. Due to rotation of the nucleus, grains in the expanding jets are swept along the azimuth of the comet giving the appearance of a set of shells, one shell per rotation. The simulated images contain 8 shells and coma dust ejected between $0-150 \mathrm{hr}$.

\subsection{Selection of size distribution of the grains in the coma and the shells}

Larger grains in the coma compared to the shells or a different grain composition for the two regions have been inferred by Hadamcik et al. (1997), Jockers et al. (1997) and Tanga et al. (1997). High polarization and high silicate IR emission (Sect. 1 and references therein) in the shells suggest predominance of smaller grains in these regions. The size distribution ' $a$ ' obtained by overall fit to coma and the shells may represent a distribution which is the average of a larger grain population for the coma and finer grains in the shells. The simulations were carried out for the distribution ' $b$ ' (Table 1), used by Hayward et al. (2000) to include larger grains. The dot dashed line in Fig. 2 shows this distribution. The size distribution of the smaller set of grains should be such that the total ensemble should produce the observed trend of increase of polarization with wavelength. As shown in the next section, the distribution ' $c$ ' with the coefficients given in Table 1 and the intensity distribution curve shown as the "dashed" line in Fig. 2 produces this trend.

\subsubsection{Effect of grain size distribution on the spatial variation of intensity, colour and polarization}

Effects of variation of porosity, organic fraction and size distributions are demonstrated in the left, middle and right columns of Fig. 3 for distributions ' $c$ ', ' $a$ ' and ' $b$ ' respectively for a few sample grain types in Table 2 . Variation of intensity, colour and polarizations along the solar - anti-solar direction across the simulated comae are shown in the lower, middle and top sections respectively. For distribution ' $c$ ', at porosities less than $45 \%$, the polarization in $0.6840 \mu, P_{\mathrm{r}}$ (solid line) is larger compared to that in $0.4550 \mu, P_{\mathrm{b}}$ (dashed line). The horizontal line at $10.0 \%$ polarization level indicates the reported value in dark regions by Hadamcik et al. (1997) in red. The lower horizontal line at $7.2 \%$ is the expected polarization in $0.4450 \mu \mathrm{m}$ based on the $P_{\mathrm{r}}-P_{\mathrm{b}}$ values from published aperture photometry. The error bars in the observed intensity cuts represent the uncertainty in the determination of sky background which is the largest source of error in the image. The upper and lower limits correspond to sky fluxes which were $0.7 \%$ and $2.4 \%$ of the central flux. The solid curve in the middle section of this panel shows the simulated colour variation. The steep fall in intensity and polarization beyond $25000 \mathrm{~km}$ on the sunward side in the coma of grains with $r_{\mathrm{m}}<2$ and distributions ' $a$ ' and ' $c$ ' occurs because of their large $\beta$ (Eqs. (4) and (5)) values. The increase in polarization beyond $35000 \mathrm{~km}$ in the sunward direction has opposite polarization colour as this is caused by the larger grains in the tail end of distribution.

Figure 3 illustrates clearly the effect of size distribution on the colour and polarization. For grains of medium porosity $(p<45 \%), P_{\mathrm{r}}>P_{\mathrm{b}}$ for distribution ' $c^{\prime}, P_{\mathrm{r}} \leq P_{\mathrm{b}}$ for ' $a$ ' and $P_{\mathrm{r}}<P_{\mathrm{b}}$ for ' $b$ '. The anti-correlation in colour and polarization as pointed out by Kolokolova et al. (2001) is clearly noticeable for ' $a$ ' and ' $b$ ': the collection ' $b$ ' (right) which has larger grains has lower polarization but larger colour than the distribution ' $a$ ' (middle). Contrary to scattering behavior of sub-micron sized grains, the absorptive grains in population ' $c$ ' are redder compared to that in ${ }^{\prime} a$ ' which contains additional larger grains. The reddening is found to increase with the refractive index of the grain either due to increase of organic fraction or reduction in porosity. It is marginally higher for Aol compared to the less absorptive SiA. As pointed out by Kolokolova et al. (2001), a grain may be redder if its size parameter $x$ is just beyond the scattering peak. The scattering peak occurs at $x \approx 2$ for absorptive grains with an effective refractive index of $\approx 1.6-i 0.2$ corresponding to $1<r_{\mathrm{m}}<2$ and $p \leq 45 \%$. For less absorptive grains of pure silicates of effective refractive index $\approx 1.4-i 0.01$ corresponding to $45 \%$ porosity, the peak occurs at $x \approx 6$ (Wickramasinghe 1973). For the average grain size of $0.24 \mu \mathrm{m}$ at the broad peak in Fig. 2 for distribution ' $c$ ', the size parameters at $0.6840 \mu \mathrm{m}$ and $0.3650 \mu \mathrm{m}$ are 2.2 and 4.4 respectively. This range in size parameter is beyond the scattering peak for absorptive grains and before for the pure silicates. This explains the red colour of the absorptive grains and the blue colour of the less absorptive ones. Further, for this distribution $P_{\mathrm{r}} \geq P_{\mathrm{b}}$, the reason for this large polarization colour is that in this domain of $0.08 \mu \mathrm{m}<s<0.6 \mu \mathrm{m}$, the grain becomes increasingly forward scattering with increase in its size parameter. Blue light is therefore more forward scattered than the red. With increase in the transmitted part of blue light which is parallelly polarized (negative polarization) the resultant polarization is lower compared to that in red. On the other hand, for the large grain population ' $b^{\prime}, P_{\mathrm{r}}<P_{\mathrm{b}}$, which is opposite to the observed trend for comets. Therefore, the difference in the grain populations in the coma and shells may in most part be due to difference in the silicate to organic ratio rather than difference in grain sizes. 

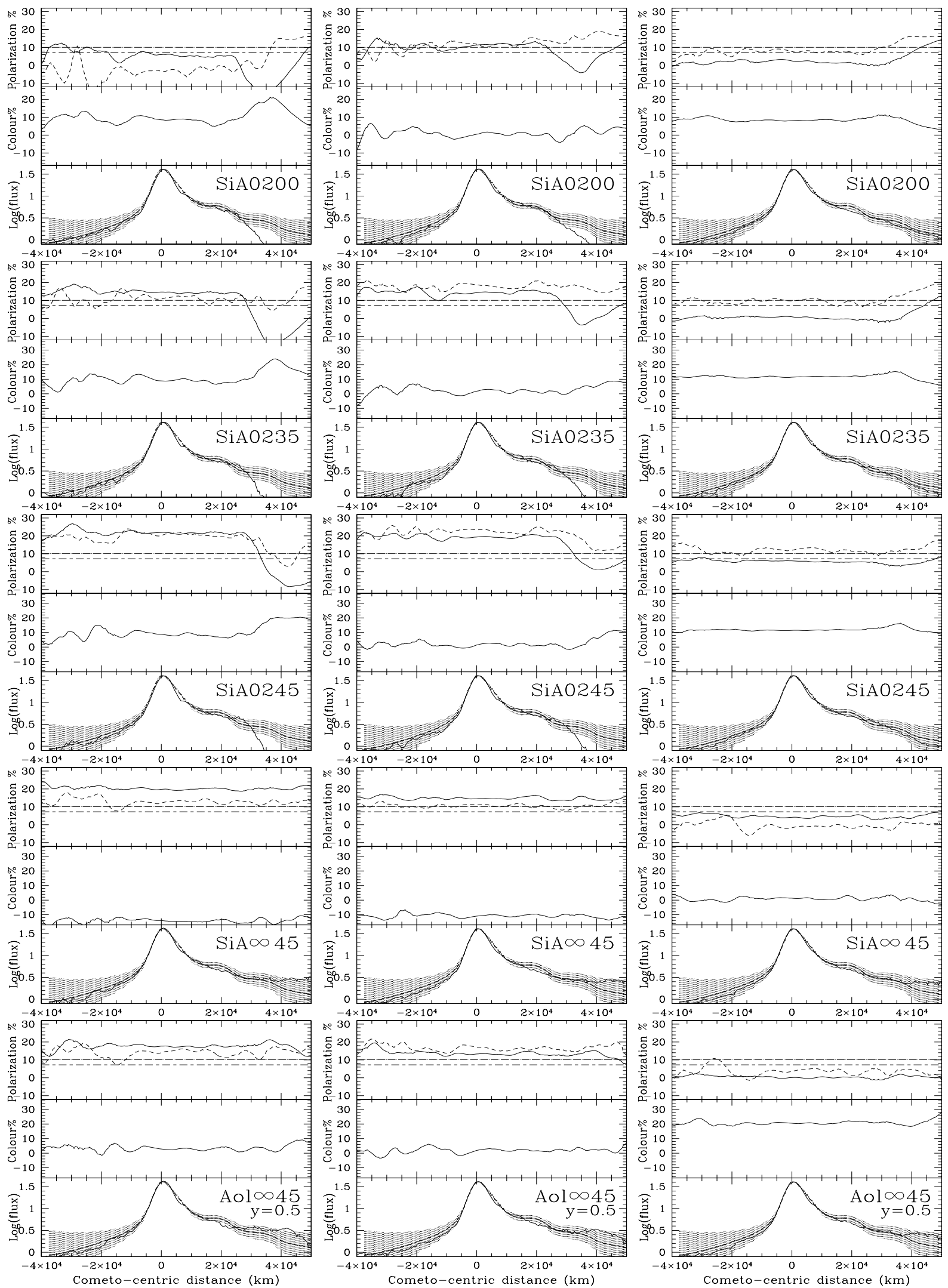

Fig. 3. Effects of grain size distribution $\left({ }^{\prime} c^{\prime}\right.$ : left, ${ }^{\prime} a^{\prime}$ : middle, ${ }^{\prime} b^{\prime}$ : right) and organic content on the spatial variation of intensity (lower panel, superposed on the observations), colour (middle panel), polarizations $P_{\mathrm{r}}$ and $P_{\mathrm{b}}$ ( solid and dashed lines in top panel), across the coma along the sunward - anti sunward direction for the geometry on April 10, 1997. The horizontal line at $10.0 \%$ polarization represents $P_{\mathrm{r}}$ in the dark regions (Hadamcik et al. 1997). The lower horizontal line at $7.2 \%$ represents the expected value of $P_{\mathrm{b}}$. 

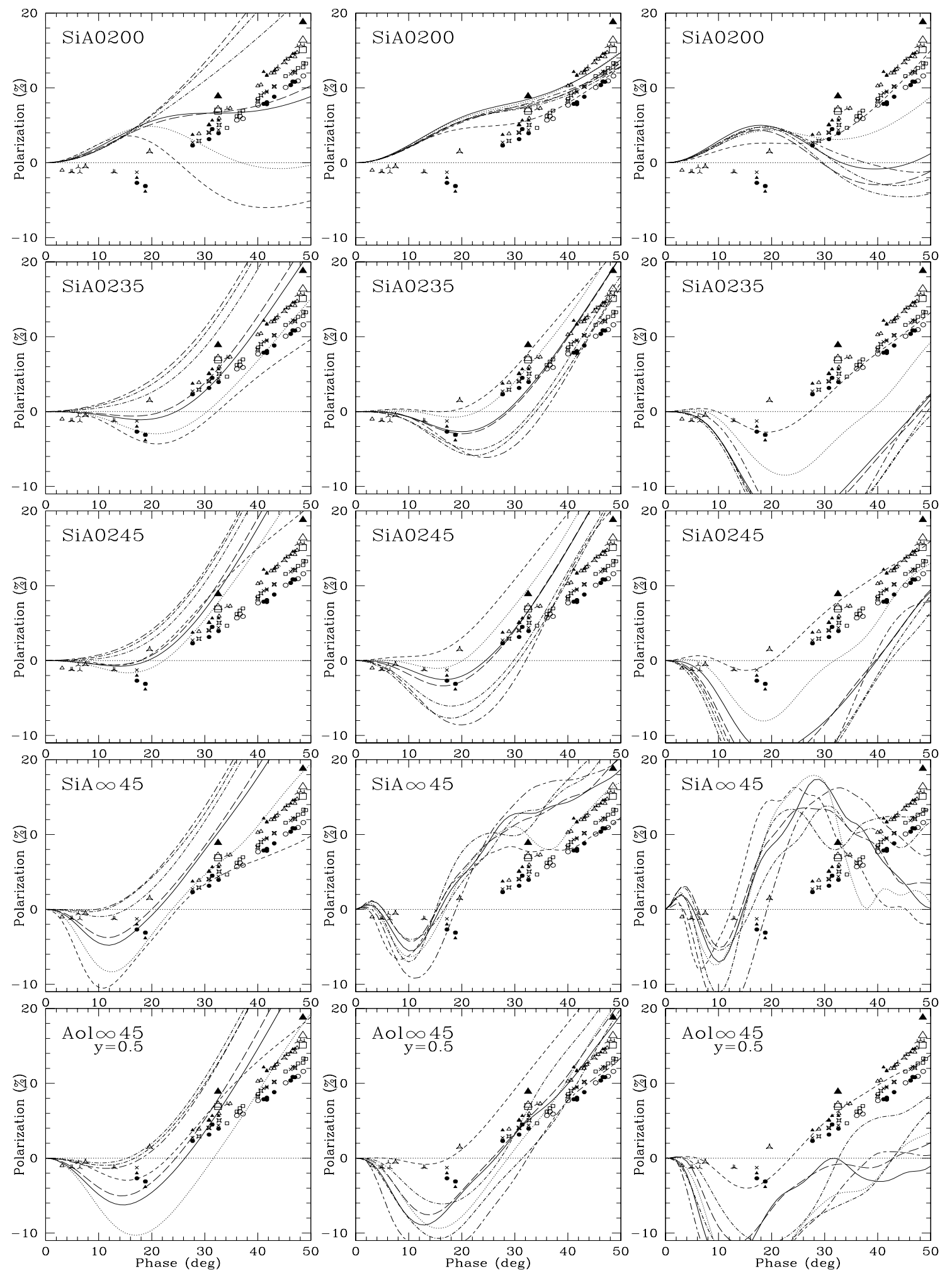

Fig. 4. Effects of grain size distribution and organic content on the phase dependence of polarization for the same sample grain types as in Fig. 3. Details of the computed curves and the data are same as for Fig. 6.

\subsubsection{Effect of grain size distribution on the variation of polarization and polarization colour with solar phase angle}

The selected grain types should also explain the phase dependent polarization in various colours. Figure 4 shows the phase dependence of the polarization produced by the same grain types as in Fig. 3 at seven continuum wavelengths for distributions $c$ (left), $a$ (middle) and $b$ (right) respectively. The polarizations at $0.3650 \mu \mathrm{m}$ (short dashed line), $0.4845 \mu \mathrm{m}$ (dotted line), $0.6840 \mu \mathrm{m}$ (solid 
line), $1.24 \mu \mathrm{m}$ (short dash - dot), $1.65 \mu \mathrm{m}$ (short dash - dot, thick line) and $2.2 \mu \mathrm{m}$ (short dash - long dash) show different trends for the three distributions. The observed polarizations in the optical by Ganesh et al. (1998), Hadamcik et al. (1997), Kiselev \& Velichko (1997) and Manset \& Bastien (1998) and the infrared observations by Hasegawa (1999) are over plotted. The phase dependence and colour of polarization again critically depend on the size distribution. The relative polarization colour represented by $\left(\left(P_{\mathrm{r}}-P_{\mathrm{b}}\right) /\left(P_{\mathrm{b}}-P_{\mathrm{u}}\right)\right)$ matches with the observed ratio for grains of distribution ' $c$ ' with $1<r_{\mathrm{m}}<2$ and $p<30 \%$, where $P_{\mathrm{u}}$ is polarization in $0.3650 \mu \mathrm{m}$. Organic refractory component has increased absorption in blue and UV. Comparing SiA grains of $45 \%$ porosity of $r_{\mathrm{m}}=2$ $(\mathrm{SiA} 0245)$ and $r_{\mathrm{m}}=\infty(\mathrm{SiA} \infty 45)$, in this grain size domain, polarizations in $0.4845 \mu \mathrm{m}$ and 0.3650 are therefore lower in the latter compared to the former because of lesser attenuation of the refracted component. Grains of Aol $\infty 45$ show their characteristic signature. The absorption in blue causes higher positive polarization in all size domains. The fluctuations in the large pure silicate grains are caused by the interference between the refracted and reflected components of light (Kolokolova et al. 1997).

Figures 3 and 4 clearly demonstrate that porosity, organic fraction and the size distribution of grains in the coma and shells play an important role in controlling the phase dependence of polarization, colour of polarization, photometric colour and spatial variation of intensity in a comet. We exploit this to investigate the composition of grains in the coma and shell separately.

\subsection{Investigation of grain materials and dust size distribution in the coma}

Synthetic comae were constructed by combining the grain types in Table 2. The relative weights were adjusted to fulfill the following four observational constrains:

1. The published values of red polarization between the shells (dark regions) of $-3.5 \%,-0.8 \%$ and $10 \%$ at phase angles of $7.5,19.6$ and 44.2 respectively by Hadamcik et al. (1997), and blue polarizations deduced from the published aperture photometry;

2. Colour of the coma in the range $10 \%-20 \%$;

3 . Higher polarization on the anti-sunward direction.

All the three size distributions were considered for each grain type. The weights and the size distributions which met this test are given in Table 3 . These weights correspond to the relative contribution to the red intensity rather than the number of grains. Thus the coma may contain a large fraction of grains with distribution ' ${ }^{\prime}$. However such grains will produce a very flattened coma. Hence these grains may be part of larger fluffy grains some of which may also be physically associated with grains of distribution ' $b$ '. The dynamics will then be controlled by an average value of $\beta$ and the coma profile will follow the observed coma (regions between the shells). Alternatively, some of the grains may have distributed sources and hence have a synthetic radial profile mimicking the grains of distribution ' $b$ '.

\subsection{Investigation of grain material in the shells}

The weights of the grain types in the shells were adjusted so that the simulated shells have the following attributes reported by Hadamcik et al. (1997), Jockers et al. (1997) and Tanga et al. (1997):

1. High polarization on the shells;

2. Bluer colour of the shells by $5 \%-8 \%$;

3. Rapid decline of polarization of the shells on the sunward side;

4. Intensity contrast between the shells and the underlying coma.

Figure 5 shows the simulations of shells made up of grain types which produce polarizations larger than the published aperture polarization measurements. The first column shows the observed processed image from VBO. The shell structures are enhanced by dividing by a synthetic image of a coma (Chakraborty 2001) in which the intensity declines as the inverse of projected cometocentric distance. The coma image was scaled so that the resultant normalized flux in the N-E edge of the image which is devoid of shells, was unity. The sets of shells expanding from the nucleus in the direction of $\mathrm{N}-\mathrm{W}, \mathrm{S}-\mathrm{W}$ and $\mathrm{S}-\mathrm{E}$ are from the sources at $-5^{\circ}, 65^{\circ}$ and $+5^{\circ}$ respectively (Papers I and II). The simulated intensity map of the shells and polarization map of the shells superposed on the coma are shown in second and third columns respectively. Each simulated image is divided into $195 \times 195$ square pixels of size $0 \prime .5$ on a side. The number density of the grains in the jets were adjusted to match the intensity variation in the shells. The fourth column shows the intensity scans of the shells superposed on the coma (lower panel), colour in $\%$ (middle panel), $P_{\mathrm{r}}$ (solid line in top panel), $P_{\mathrm{b}}$ (short dashed line in top panel) and $P_{\mathrm{r}}-P_{\mathrm{b}}$ (long dashed line in top panel). Each grain type has its characteristic signature in the intensity, colour and polarization map.

1. Row 1 (starting from below): grains of the type "Si $\infty 45$ " with distribution ' $a$ ' produce shells that resemble the observed ones. The shells are bluer but the polarization is only marginally higher than the ambient coma.

2. Row 2: grains of the type "Si $\infty 45$ " but with distribution ' $c$ ' appear to be likely candidate as $P_{\mathrm{r}}$ is larger on the shell compared to coma. However neither is there a rapid fall in polarization in the sun ward direction nor an increase in polarization in the anti sunward direction.

3. Row $3 \&$ 4: increasing the organic fraction to $r_{\mathrm{m}}=$ 8 \& 12 causes the older shells to be depleted of finer grains as these are blown away towards the antisunward direction. The shells are compressed and a piling up of shells older than four rotations causes a "shell pause" like structure in the polarization map 

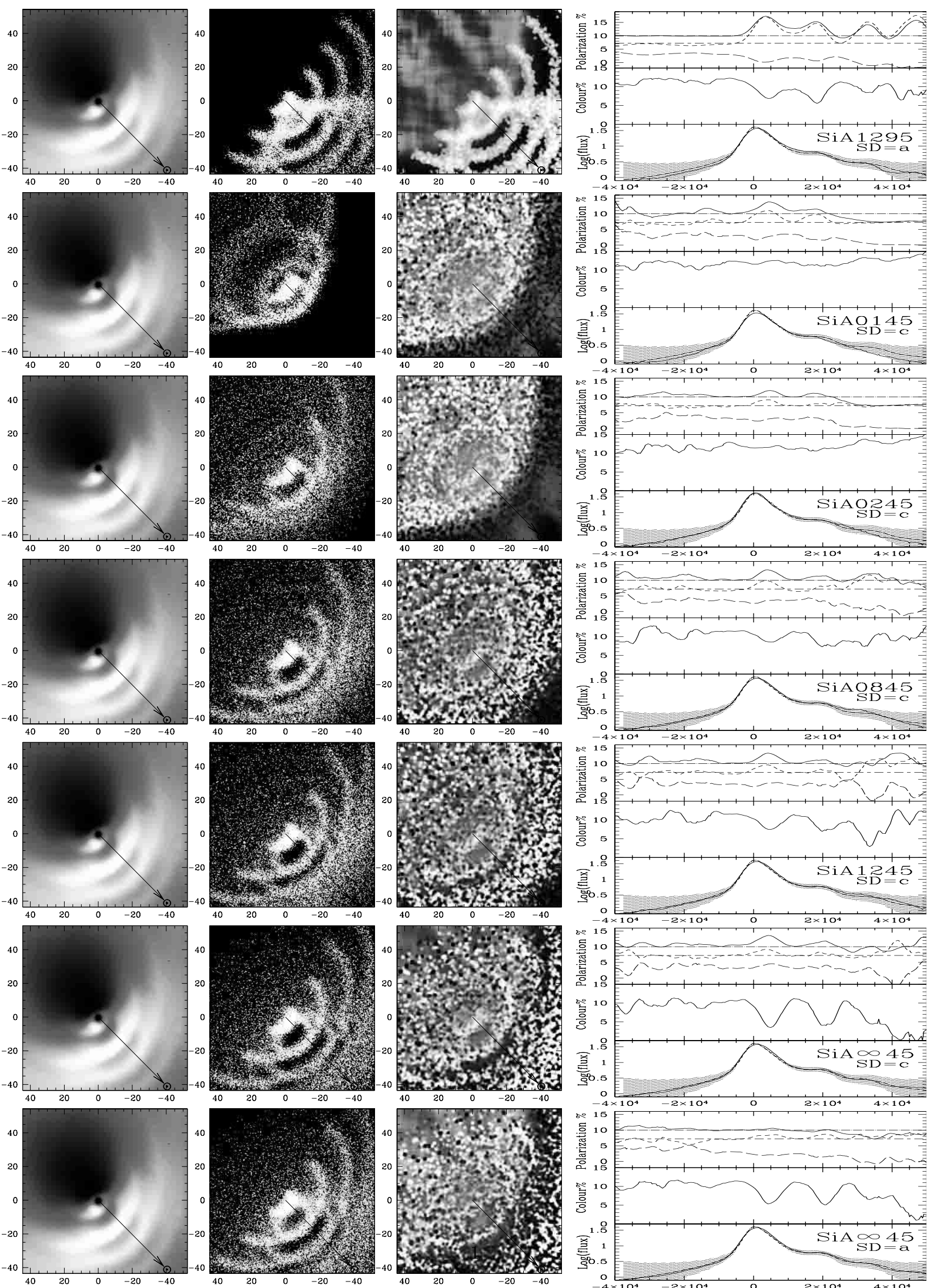

Fig. 5. Column 1: observed processed image from VBO $\times$ (projected cometocentric distance $)^{-1}$. North is top and East is left. The sets of shells expanding from the nucleus in the direction of $\mathrm{N}-\mathrm{W}, \mathrm{S}-\mathrm{W}$ and $\mathrm{S}-\mathrm{E}$ are from the sources at $-5^{\circ}, 65^{\circ}$ and $+5^{\circ}$ respectively. Columns 2 and 3: simulated intensity (shell) and polarization (shell + coma) maps. Column 4: intensity scans of the shells superposed on the coma (lower panel), colour in \% (middle panel), $P_{\mathrm{r}}$ (solid line in top panel), $P_{\mathrm{b}}$ (short dashed line in top panel) and $P_{\mathrm{r}}-P_{\mathrm{b}}$ (long dashed line in top panel). 
Table 3. Possible grain types in the coma and shells.

\begin{tabular}{|c|c|c|c|c|c|c|}
\hline \multirow{3}{*}{$\begin{array}{c}\text { Contribution: } \\
m_{\text {sil }} / m_{\text {org }}: \\
\text { Porosity: } \\
\text { Silicate Type: }\end{array}$} & \multicolumn{4}{|c|}{ Coma } & \multicolumn{2}{|c|}{ Shell } \\
\hline & \multirow{2}{*}{$\begin{array}{c}1 \leq \\
0 \% \leq p \leq 50 \% \\
\text { SiA } / \mathrm{SiX}\end{array}$} & \multirow{2}{*}{$\begin{array}{c}\mathrm{m} \leq 2 \\
40 \% \leq p \leq 50 \% \\
\mathrm{SiA} / \mathrm{SiX}\end{array}$} & \multicolumn{2}{|c|}{$\begin{array}{c}8 \leq r_{\mathrm{m}} \leq \infty \\
30 \% \leq p \leq 50 \%\end{array}$} & \multirow{2}{*}{$\begin{array}{c}8 \leq r_{\mathrm{m}} \leq \infty \\
40 \% \leq p \leq 50 \% \\
\mathrm{SiA}\end{array}$} & \multirow{2}{*}{$\begin{array}{c}1 \leq r_{\mathrm{m}} \leq \infty \\
p \geq 90 \% \\
\mathrm{SiA} / \mathrm{SiX}\end{array}$} \\
\hline & & & $\mathrm{SiA} / \mathrm{SiX}$ & $\mathrm{Aol}^{1}$ & & \\
\hline $\begin{array}{l}\text { Fractional weight }^{2} \text { : } \\
\text { Size Distribution: }\end{array}$ & $\begin{array}{c}0.47 \pm 0.05 \\
c\end{array}$ & $\begin{array}{c}0.14 \pm 0.05 \\
b\end{array}$ & $\begin{array}{c}0.05 \pm 0.05 \\
c\end{array}$ & $\begin{array}{c}0.14 \pm 0.05 \\
b\end{array}$ & $\begin{array}{c}0.12 \pm 0.02 \\
c\end{array}$ & $\begin{array}{c}0.06 \pm 0.02 \\
a\end{array}$ \\
\hline
\end{tabular}

1. Weight near perihelion.

2. Relative contribution to intensity in red.

and an abrupt decline in polarization. In the intensity map, the pile up of matter merges with the coma. This region appears bluer in the colour map because of the shell material. For a detailed comparison with observed colour and polarization maps, shells ejected over a larger numbers of the nuclear rotations $(>8)$ and coma ejections over an extended duration $(t>150 \mathrm{hr})$ are required.

4. Row 5 \& 6: with increase in the organic fraction to $r_{\mathrm{m}} \leq 2$, the radiation pressure blows out grains from the shells older than two rotations and produces a very steep increase in polarization in the anti-sunward direction and an abrupt fall in the sunward direction. The contrast in colour between the coma and the shells disappears The effect is pronounced for $r_{\mathrm{m}}=1$.

5. Row 7: porous grains are known to produce large polarizations. Hence we considered a collection of highly porous grains with distribution ' $a$ ' which are assumed to be homogeneous in the Rayleigh limit. This set produces well constrained shells which survive several rotation periods. This is because of lower $\beta$ values which vary slowly with the grain size. Although the present approach of EMT + Mie theory is known to have serious limitations for porous grains, these simulations help to visualize the signature of highly porous homogeneous grains. The streaks in the polarization map streaming away in the anti-sun direction represent the small grains in the population.

From Fig. 5, it is apparent that larger polarization and bluer colour on the shells are produced by grains of $\mathrm{SiA}$ with $r_{\mathrm{m}}>8$. However, not more than 3 shells survive the radiation pressure forces while the observed image after careful evaluation and removal of the underlying coma shows up to 8 shells (Chakraborty 2001). Therefore a population of grains with high polarization but low $\beta$, similar to the over simplified example of a uniform porous grain in Row 7 in Fig. 5 also appear to be needed to explain the survival of older shells. A reliable estimate of the fractional weights of these grain types can only be made by a quantitative comparison of the polarization maps. In the absence of availability of such a data set, we make use of the aperture polarimetry observations. As the aperture photometry represents combined contribution of coma and shells, the grains which may explain the polarization on the shell, when added in the same proportion to the selected combination representing the coma should explain the aperture polarimetric data. The best fitting weights for the shells which fitted polarization vs. phase curves are given in Table 3. Figure 6 shows the resultant simulated polarization vs. phase curves. At phase angles larger than $30^{\circ}$, the fits were better if the relative fraction of $\mathrm{Mg}$ poor silicate grains with distribution $b$ were increased gradually with heliocentric distance. A possible explanation for this is given in Sect. 6 .

The resultant simulated intensity maps of the shells and polarization maps of shells and coma using weights in Table 3 are shown in the top, middle and right panels of Fig. 7. The first panel is the processed observed image as in Fig. 5. Separation of the high $\beta(p=45 \%)$ grains and low $\beta$ grains $(p=95 \%)$ components produces the splitting of the shells in the $\mathrm{N}-\mathrm{W}$ and $\mathrm{S}-\mathrm{E}$ region of the simulated image (Table 3).

The lower panel of Fig. 7 shows the intensity, colour and polarization scans in the sunward - anti-sunward direction. In order to reduce the fluctuations arising due to discretization of cometary longitudes and latitudes, the scans have been averaged over 9 pixels (4."5). The polarization contrast between the coma and the shells are therefore reduced in these plots. The maximum and minimum value of polarization in the map however correspond to $16 \%$ in the bright regions and $2 \%$ beyond the 4 th shell in the sunward direction.

\subsection{Sources of errors and limitations of the present technique}

The width and separation of the shells depend on the pole position, velocity $v_{\text {gr }}$ and the acceleration $\alpha$ of the grains. The later two quantities depend on the size distribution and the nature of the grains. For the geometry on 10 April, 1997, the uncertainty in the computed separation of the shells from the source at $65^{\circ}$, is found to be $\pm 10 \%$ along the projected sunward direction for a change in $\alpha_{\mathrm{p}}$ by $\pm 15^{\circ}$ and $\delta_{\mathrm{p}}$ by $\pm 10^{\circ}$. The model thus reproduces the shells within an accuracy of $\pm 5 \%$ in the shell separation corresponding to the uncertainty of $\pm 5^{\circ}$ in the pole position used in the present study following Paper II. The grain velocities cannot therefore be constrained 


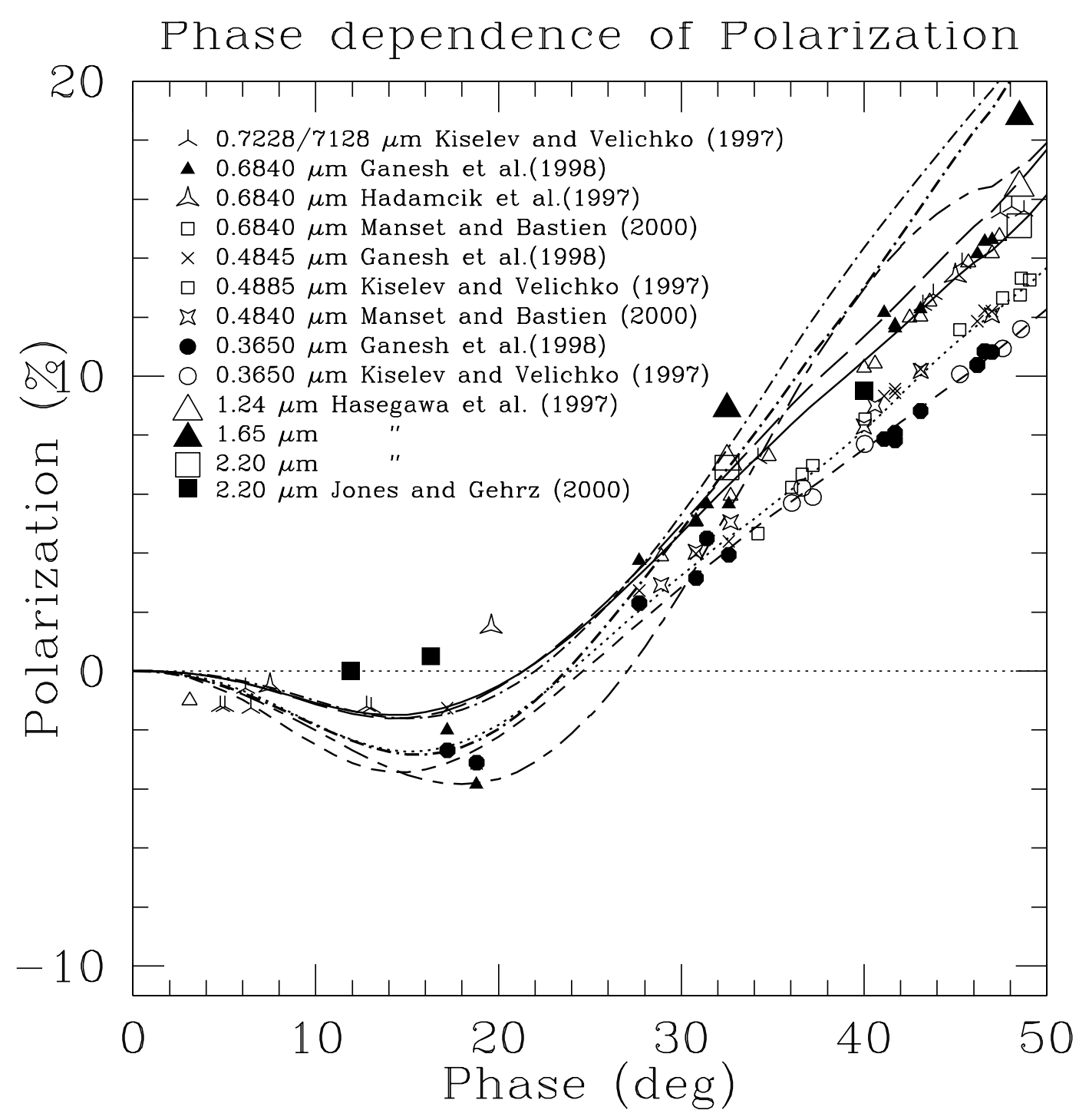

Fig. 6. Fitted polarization vs. phase angle curves for comet Hale-Bopp: computed curves: $0.6840 \mu \mathrm{m}$ (solid line), $0.4845 \mu \mathrm{m}$ (dotted line), $0.3650 \mu \mathrm{m}$ (dashed line), $0.7200 \mu \mathrm{m}$ (long dashed line), $1.24 \mu \mathrm{m}$ (short dash - dot, thin line), $1.65 \mu \mathrm{m}$ (short dash - dot, thick line) and $2.20 \mu \mathrm{m}$ (long dash - short dash).

better than $\pm 5 \%$. For a more rigorous check on the derived pole positions and the grain velocities, intensity scans across the observed image along the directions 184.5 (b), 194.5 (c), 214.5 (d), 224.5 (e, the sunward direction), 234.5 (f), 244.5 (g) and 254.5 (h), divided by the scans along the same directions across the simulated coma of composition in Table 3 are compared with the simulated scans in Fig. 8 (lower panel). The directions are marked on the observed image in the top panel. The nucleus rotated by $154^{\circ}$ during the interval of time between the ejections of the two extreme sections of the shells. The cuts along the directions $145^{\circ} .5$ (a) and $285^{\circ} .5$ (i) are across the equatorial shells. The location and width of the shells match reasonably well over a range of hour angle of the Sun spanning $154^{\circ}$ for the high latitude shells as well as the two cuts across the equatorial shells. The cuts along the directions ' $b$ ', ' $c$ ' and ' $h$ ' are contaminated by the equatorial shells. For all the cuts, at distances larger than $30^{\prime \prime}-35^{\prime \prime}$, deviations between the observed and simulated cuts become noticeable. This may be attributed to low intensity level of the observed image at these distances as a result of which the errors associated with improper sky subtraction increase rapidly, as indicated by the large error bars in Figs. 5 and 7. The simulated image also suffers from limitations due to under-sampling of the shells of only 8 rotations and coma of 13 rotations. The deviations may also be due to the simplicity of the model which does not take into account the grain evolution processes like evaporation or splitting. These processes alter $\beta$ of the grains and hence their dynamics. There is also a gradual increase in the intensity of the first shell from morning till evening (a-i). This may be due to the assumption of uniform dust emission from dawn till dusk made in the simulation of the coma, which is used to normalize the intensity. The simulated "shell+coma" profile may be unaffected because of similar assumptions for simulating the shells. The local 

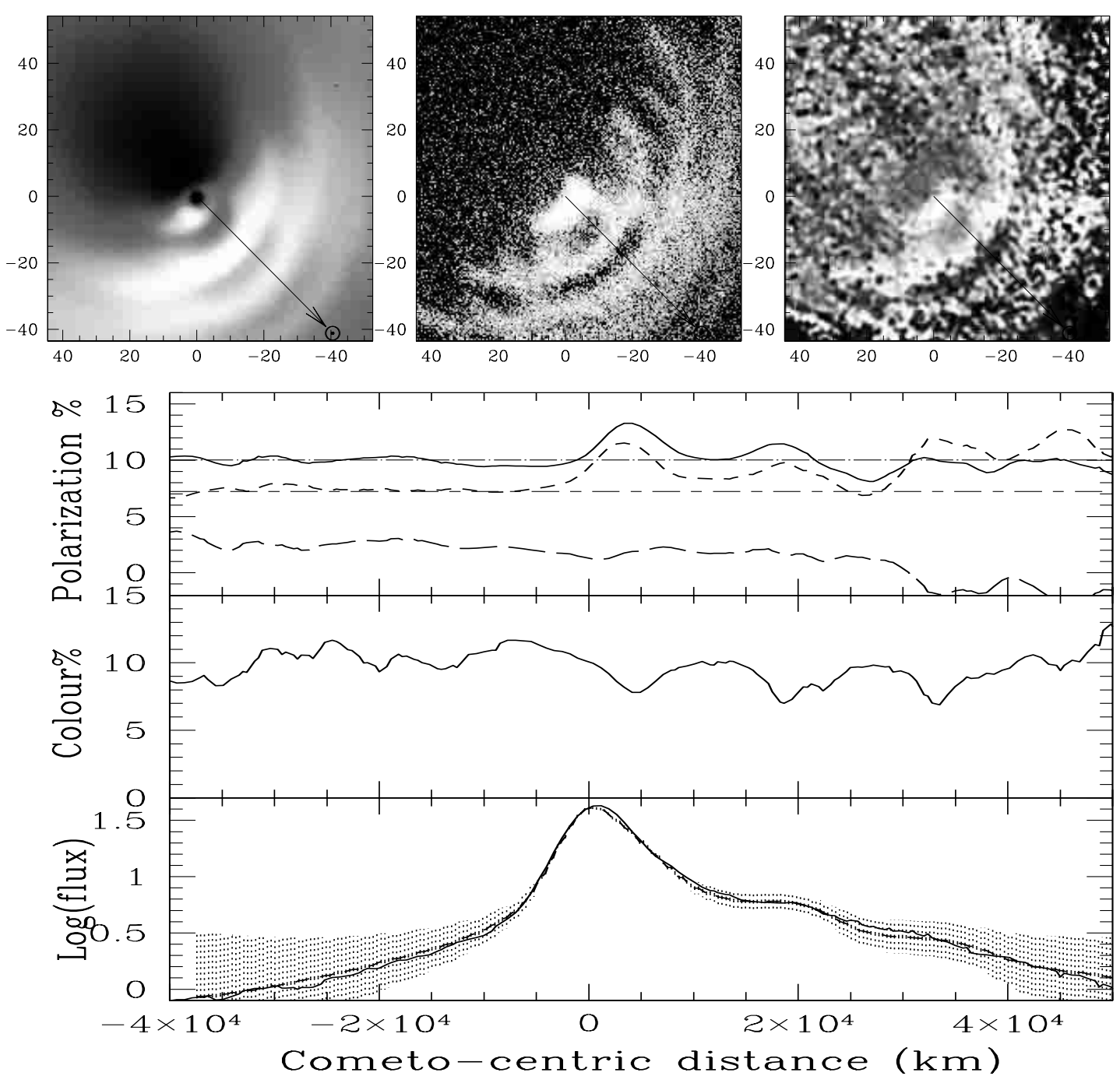

Fig. 7. Top left: the processed observed image as in Fig. 5. Top middle and right: simulated intensity maps of the shells and polarization maps of shells+coma using weights in Table 3. The lower panel shows the intensity, colour and polarization scans in the sun-ward - anti-sun-ward direction.

$E-W$ asymmetry appears to smoothen out in the outer regions. In any case, inferring the grain composition by matching the observed separation and width of the first few shells alone will be questionable because a variety of factors such as grain size distribution, spread in porosity and organic content of the grains determine the average value and spread in $\beta$ of the ensemble. Fortunately, these factors influence the colour and the phase dependence of polarization at different wavelengths in different ways (Figs. 3 and 4). Hence as attempted in the present investigation, the spatial match must be accompanied by comparisons of (1) colour and (2) polarization of the observed and simulated shells (Figs. 3 and 5). The derived range in porosity and organic fraction must explain the published phase curves in three colours (Fig. 6). This places further constraints on the derived values of $p$ and $r_{\mathrm{m}}$. However, there are limitations in the present model which should not be overlooked. As explained in the following sections, Mie theory does not accurately predict the polarization vs. phase curves of porous grains which are not homogeneous.
Lack of our understanding of the nature of the organic component limits the reliability of the derived compositions. If the composition of the organic material assumed in Sect. 4.2 is changed by including the extremely reddish material inferred by Tegler and Romanishin (2000) in the Kuiper-belt objects, the fitted fraction of Aol will be modified. This may also change the best fitting size distribution as this parameter was constrained to explain the colour of the grains. Further, despite the availability of an extensive and wide range of data set on this comet, the inclusion of a large parameter space will hinder in getting a unique solution due to cross talk between the effects of different parameters.

For reasons explained in Sect. 4.2, the present technique cannot be used to identify the mineralogy of the silicates in the grains although distinction can be made between the low and high $\mathrm{Mg}$ content silicates based on the photometric and polarization colours (Figs. 3 and 4). Such investigations are best made using IR spectroscopy (Hayward et al. 2000; Wooden et al. 1999). However, the 

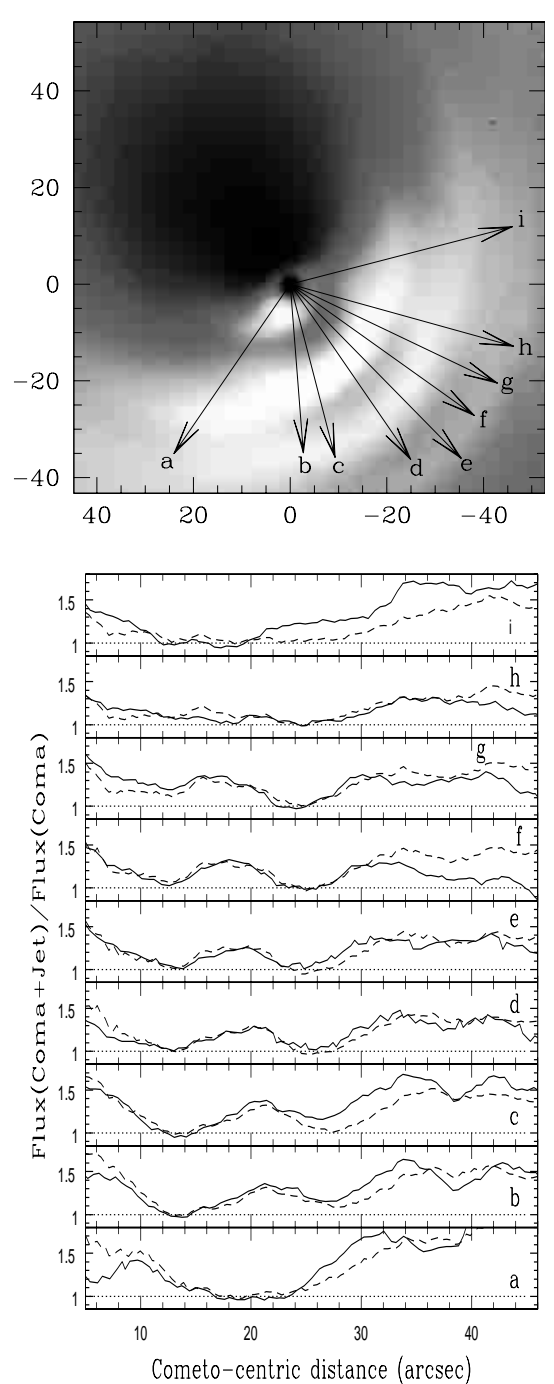

Fig. 8. Top: the processed observed image as in Fig. 5 showing the directions of scans. Lower panel: intensity scans across the observed (dashed line) and simulated (solid line) images along directions $145^{\circ} .5$ a), $184^{\circ} .5$ b), $194^{\circ} .5$ c), 214.5 d), 224.5 e), $234^{\circ} .5$ f), $244^{\circ} .5$ g), $254^{\circ} .5$ h) and $285^{\circ}$ i), divided by the scans along the same directions across the simulated coma of composition in Table 3 .

derived porosities and organic contents in the present work can help in constraining the IR continuum, and hence the two techniques are complementary to one another.

\section{Polarization vs. phase angle curves in NIR}

The simulations at $1.24 \mu \mathrm{m}, 1.65 \mu \mathrm{m}$ and $2.2 \mu \mathrm{m}$ are shown in Fig. 6 along with the observations by Hasegawa et al. (1999) and Jones \& Gehrz (2000). Except near $30^{\circ}$ phase, the fitted curves do not match very well with these high quality observations. The possible reasons could be any of the following: the narrow size range population of grains in Table 3 which comprise of $61 \%$ in the coma and $12 \%$ in the shell sampled by the aperture may not be isolated but may be sub units of highly porous larger grains with porosity $p>90 \%$ as suggested by
Kolokolova et al. (2001), Levasseur-Regourd et al. (1997) and Xing \& Hanner (1997). Such a grain model is similar to the ballistic cluster aggregation (BCCA) investigated by Mukai et al. (1992). At these porosities, the individual units will interact independently with the incoming visible light and will act as isolated scatterers (Xing \& Hanner 1997). However, at infrared wavelengths, the separation between the sub units becomes significant and the porous grain should be treated as an aggregate with non Rayleigh inclusions of these sub units. For such a study the present method of EMT and Mie theory is inadequate and the more elegant technique of Discrete Dipole Approximation (DDA) using the code DDSCAT by Draine \& Flatau (1994) will be appropriate. Wolf et al. (1998) compared the results using different EMT rules along with Mie scattering with that using the technique of Discrete Dipole Approximation and showed that significant differences are noticed for grains with non Rayleigh inclusions. In particular, these authors point out that for such grains, computations using DDA reduce forward scattering and the polarization phase function becomes distinctively positive compared to Rayleigh inclusions and the EMT solutions. Large negative polarization at low phase angles in Fig. 6 may significantly reduce if DDA is used. Further, the present method treats the small grain population as individual grains. In the $1.25 \mu \mathrm{m}-2.2 \mu \mathrm{m}$ region, these will scatter poorly and the signature of the larger grain population ' $b$ ' dictates the resultant polarization at these wavelengths. The present investigation restricted the fit to polarization in the optical region only. Extension to polarization in the $J H K$ bands are important because it will help in investigating the crystal field band of $\mathrm{Fe}^{2+}$ in the $1.1 \mu \mathrm{m}$ region of Fe rich olivines. (Dorschner et al. 1995). This band is week in Mg poor pyroxene. The effect of this band which is spread over the JHK bands will be to increase the opacity of the grains and hence the polarization. A detailed fit from UV to $J H K$ using DDA is planned as the next phase of investigations.

\section{Possible dichotomy in the surface composition of comet Hale-Bopp}

The relative fraction of $\mathrm{Mg}$ poor silicate grains needed to be increased gradually with heliocentric distance/solar phase angle in order to match the polarization colour at phase angles larger than $30^{\circ}$. Presence of the component Aol with size distribution $b$ in the ensemble causes an increased absorption (Dorschner et al. 1995) and hence an increase in positive polarization at $0.3650 \mu \mathrm{m}$ compared to $0.4845 \mu \mathrm{m}$ and longer wavelengths (Fig. 4). The same effect can be mimicked if the organic part of the grain resembles the very red material found in the Kuiper-belt objects (Tegler \& Romanishin 2000) instead of the composition of organic refractory and amorphous carbon of equal mass assumed in the present work (Sect. 4.2). Organic refractory (Li \& Greenberg 1997) has a gradual increase in $k$ with decreasing wavelength in the optical region, while for amorphous carbon (Rouleau \& Martin 1991) $k$ although 
large, decreases with decreasing wavelength. As very little is known about the nature of cometary organics, any change in the assumed composition of the organic component may also alter the shape of the polarization curve and hence the derived compositions. Caution is also needed in interpreting the required change in the composition of $\mathrm{Aol}$ (or grains with enhanced blue absorption) with heliocentric distance/phase angles for a good fit, as a real effect. This unreliability arises due to the use of Mie scattering and Effective Medium Theory in our model which has been shown to be inadequate in reproducing the polarization vs. phase curves for porous grains of non Rayleigh inclusions (Wolf et al. 1998). As naturally occurring composite grains are more likely to have non Rayleigh inclusions than to be of homogeneous composition, the results presented here may be substantially improved if DDA is used. On the other hand, due to changes in solar illumination geometry during the apparition of the comet, there is also reason to suspect that the requirement of change in composition may at least be partially real due to possible dichotomy in the surface composition in the northern and southern regions of comet Hale-Bopp. The latter regions being sunlit during and prior to 1996 and the former regions in 1997. The porcupine like appearance of the jets (O'Meara et al. 1996) have been explained due to dust emission from discrete southern sources at latitudes of $-70^{\circ},-35^{\circ}$ and $0^{\circ}$ by Sekanina \& Boehnhardt (1998) and of $-65^{\circ},-35^{\circ}$ and $\pm 5^{\circ}$ by Vasundhara \& Chakraborty (1998). The prominent shell structures near perihelion have been attributed by these authors to a source near $60^{\circ}$ latitude. As shown in the top panel of Fig. 9, the solar phase angle while oscillating between oppositions steadily increased with heliocentric distance. The dates of the available polarimetric observations in red are marked with the same symbols as in Figs. 4 and 6. Variation of the angle $B^{\prime}$ (Sect. 2.2) with heliocentric distance is shown in the middle panel. The activity of the sources near $-65^{\circ}$ and $+65^{\circ}$ as determined by the average value of the cosine of the local zenith distance of the Sun over a rotation period of the comet, $\left\langle\cos z_{\odot}\right\rangle$, at different heliocentric distances are shown as solid and dashed lines respectively in the lower most panel. The activity of the latter source increased steeply during February 1997. The best fit to polarization vs. phase curves corresponds to a 15 fold increase in the relative number of $\mathrm{Mg}$ poor grains at the phase angle of $48^{\circ}$ compared to their composition at $<30^{\circ}$.

The proposed dichotomy may also explain the presence of the most volatile gaseous species to be more abundant at large heliocentric distances before perihelion reported by Biver et al. (1997). The stalling or reduction of production rates between 3 and 1.6 AU before perihelion reported by them coincides with the epoch when the solar illumination in the southern regions started decreasing rapidly. Wooden et al. (1999) note that the $9.3 \mu \mathrm{m}$ shoulder in the 7.5-13.4 IR spectra of the comet is present only in the images when the heliocentric distances were less than 1.2 AU. From Fig. 9 it is seen that the activity of the source at $+65^{\circ}$ increased steeply at heliocentric distances

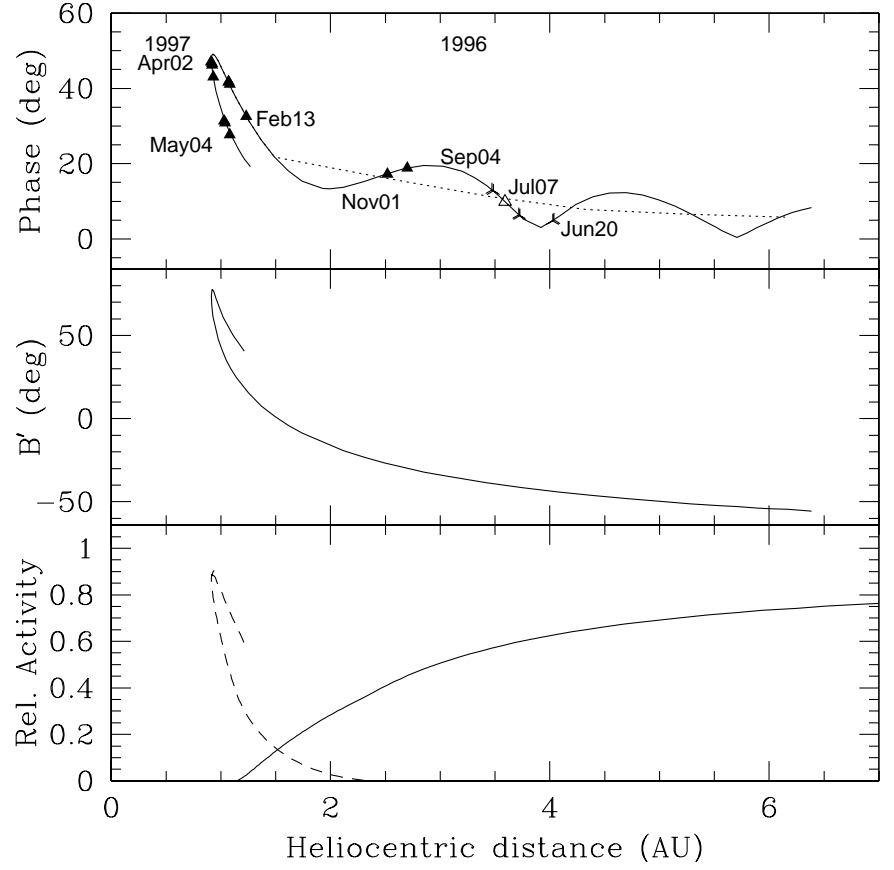

Fig. 9. Top panel: variation of the solar phase angle with heliocentric distance. The dates of the available polarimetric observations in red are marked with the same symbols as in Fig. 6. Middle panel: variation of the angle $B^{\prime}$ (Sect. 2.2) with heliocentric distance. Lower panel: the activity of the sources near $-65^{\circ}$ (solid line) and $+65^{\circ}$ (dashed line) as determined by the average value of the cosine of the local zenith distance of the Sun over a rotation period of the comet, $\left\langle\cos z_{\odot}\right\rangle$.

closer than $\approx 1.2 \mathrm{AU}$, hence the extra feature at $9.3 \mu \mathrm{m}$ may have come from the northern region of the comet.

\section{Conclusions and scope for further improvements}

A model has been described to obtain intensity and polarization maps of coma and shell (jet) of comets. The intensity profile across the simulated coma and shells for the geometry on 10 April, 1997 of comet Hale-Bopp is compared with the observed profile. Published values of polarization and colour in the coma and shells of comet Hale-Bopp on a close by date are used to investigate the nature of the grains, their organic content and porosity. Published aperture photometry at $0.6840 \mu \mathrm{m}, 0.4845 \mu \mathrm{m}$ and $0.3650 \mu \mathrm{m}$ are initially used to short-list the possible range in porosities and the silicate to organic mass ratio. The relative weights of grain types in the coma and shell are further constrained such that the combined coma and shell population 1) reproduces the polarization vs. phase curve, 2) explains the high polarization on the shells and 3) their bluer colour (compared to coma). The results indicate that although it is impossible to determine the nature of the silicate (pyroxenes/olivines or amorphous/crystalline) using the present 
technique, the porosity, organic fraction and size distribution of the grains can be constrained. The scattered light from coma has polarization and colour characteristics which point to presence of at least $60 \% \pm 5 \%$ of grains with $1 \leq m_{\mathrm{sil}} / m_{\mathrm{org}} \leq 2$ and of medium porosity in the range $0-50 \%$. A narrow size distribution between $0.08 \mu \mathrm{m}-0.6 \mu \mathrm{m}$ is inferred for $70 \%$ of the grains in the coma and shell. These grains could be the building blocks of larger fluffy grains of porosity larger than $90 \%$. At these porosities the individual units will interact independently with the incoming light and will act as isolated scatterers (Xing \& Hanner 1997). The silicate in these grains could be Astronomical silicate (SiA) or a combination of $\mathrm{Mg}$ poor and $\mathrm{Mg}$ rich olivines or pyroxenes in crystalline or amorphous state. The shells of comet Hale-Bopp which produce larger polarization and are bluer compared to the coma appear to contain grains of silicate with lower organic fraction $\left(r_{\mathrm{m}} \geq 8\right)$ and of moderate porosity $(30-50 \%)$. In order to explain the presence of as many as 8-10 shells in well processed images, the fit using the present dynamical model imposes the condition that the shells must contain grains of $\beta<1$. This is independent of detailed assumptions on the optical properties of the grains or the scattering theory. Our Mie scattering calculations using Effective Median Theory to calculate the overall optical properties suggest that highly porous grains with $p \geq 90 \%$ made up of sub units in the form of Rayleigh inclusions (uniform density) have this property. This inference can be tested or further refined by using more elegant light scattering theories or techniques as mentioned below. Alternatively, the fluffy grains made of sub micron grains as sub units may also contain larger grains which may control the dynamics by reducing the effective $\beta$. The change in slope of the polarization $v s$ phase curves at high phase angles which occurred at closer heliocentric distances and when the northern region of the comet was sunlit, could be better explained by increasing the fraction of the $\mathrm{Mg}$ poor olivine grains with $r_{\mathrm{m}} \geq 12$ at these epochs. This may be due to the limitations of the Mie theory in constructing the phase vs. polarization of natural grains. Considering the geometry of the apparition of the comet, a dichotomy in the surface composition of the comet may also be responsible to some extent for such an enhancement of these grain types required to fit the data set near perihelion.

In spite of the limitations of EMT theory with Mie solutions, the present results which indicate predominance of grains in the size range $0.08 \mu \mathrm{m}-0.6 \mu \mathrm{m}$, and porosity in the range $0-45 \%$ are similar to that suggested by Xing \& Hanner (1997) using DDSCAT. They find that a mixture of aggregates of carbonaceous and silicate monomers of a tenth of micron in size and of intermediate porosity of $60 \%$ resembles the observed phase dependence of polarization of comets. For this intermediate porosity, it therefore appears that, at least for the polydisperse grains, EMT solutions may approximate the real scattering solutions. The DDA technique and assuming the grains to be made up of organic coated silicate monomers (Greenberg
\& Hage 1990) will be attempted as the next step to match the polarizations in the range from $0.3650 \mu \mathrm{m}$ to $2.2 \mu \mathrm{m}$. The use of computational techniques can be dispensed with and one could directly use (1) the microwave and laser facilities to determine scattering and colour signatures related to the physical properties of dust grains (Weiss-Wrana et al. 1985a, 1985b), (2) the database created by Gustafson et al. (1999) using microwave analogues of complex grain systems or (3) the data from the scattering experiments (PROGRA) conducted under micro gravity conditions on fluffy grains (Levasseur-Regourd et al. 1998; Worms et al. 1999) with optical constants similar to Astronomical silicate. In any case, by adapting a superior light scattering theory or using results of laboratory simulations, the present model can be used to fully extract the information available from polarization and intensity maps of cometary shells and coma.

Acknowledgements. I am grateful to Dr. P. Chakraborty of the Indian Institute of Astrophysics for the processed image of 10 April, 1997 and access to his software to convert the simulated images to IRAF images. I am also grateful to Dr. A. Li for the optical constants of the silicates. I am indebted to the referee for the valuable comments.

\section{References}

Bellucci, G. 1998, A\&A, 333, 369

Biver, N., Bockelée-Morvan, D., Calon, P., et al. 1997, Earth, Moon, and Planets, 78, 5

Bohren, C. F., \& Huffman, D. R. 1983, Absorption and scattering of light by small Particles (John Willey \& Sons Inc) Chakraborty, P. 2001, Ph.D. Thesis

Crovisier, J., Leech, K., Bockelée-Morvan, D., et al. 1997, Science, 275, 1904

Dorschner, J., Begemann, B., Henning, et al. 1995, A\&A, 300, 503

Draine, B. T. 1985, ApJS, 57, 587

Draine, B. T., \& Flatau, P. J. 1994, J. Opt. Soc. Am. A, 22, 1491

Draine, B. T., \& Lee, H. M. 1984, ApJ, 285, 89

Finson, M. L., \& Probstein, R. F. 1968, ApJ, 154, 327

Fulle, M. 1987, A\&A, 171, 327

Furusho, R., Suzuki, B., Yamamoto, N., et al. 1999, PASJ, 51, 367

Galdemard, P., Lagage, P. O., Dubreuil, D., et al. 1997, Earth, Moon, and Planets, 78, 271

Ganesh, S., Joshi, U. C., Baliyan, K. S., \& Deshpande, M. R. 1998, A\&A, 129, 489

Greenberg, J. M., \& Hage, J. I. 1990, ApJ, 361, 260

Gurnette, B. L., \& Woolley, R. v. d. R. (eds.) 1960, in Explanatory Supplement to the Astronomical Ephemeris and The American Ephemeris \& Nautical Almanac, prepared by the Nautical Almanac Offices, UK \& USA, 342

Gustafson, B. A. S. 1999, in Formation and Evolution of Solids in Space, ed. J. Mayo Greenberg, \& Li. Aigen (Kluwer Academic Publishers), 535

Hadamcik, E., Levasseur-Regourd, A. C., \& Renard, J. B. 1997, Earth, Moon, and Planets, 78, 365 
Hage, J. I., \& Greenberg, J. M. 1990, ApJ, 361, 251

Hanner, M. S. 1985, Adv. Space. Res., 4(9), 189

Hasegawa, H., Ichikawa, T., Abe, S., et al. 1997, Earth, Moon, and Planets, 78, 353

Hayward, T. L., \& Hanner 1997, Earth, Moon, and Planets, 78,265

Hayward, T. L., Hanner, M. S., \& Sekanina, Z. 2000, ApJ, 538, 428

Jewitt, D., \& Meech, K. J. 1986, ApJ, 310, 937

Jockers, K., Rosenbush, V. K., Bonev, T., \& Credner, T. 1997, Earth, Moon, and Planets, 78, 373

Jones, T. J., \& Gehrz, R. D. 2000, Icarus, 143, 338

Jorda, L., Rembor, K., Lecacheux, J., et al. 1997-1999, Earth, Moon, and Planets, 77, 167

Kiselev, N. N., \& Velichko, F. P. 1997, Earth, Moon, and Planets, 78, 347

Kolokolova, L., Jockers, K., \& Chernova, G. 1997, Icarus, 126, 351

Kolokolova, L., Gustafson, B. A. S., Jockers, K., \& Lichtenberg, G. 2001, J. Geophys. Res., 106, 10113

Krishna Swamy, K. S., \& Shah, G. A. 1987, Earth, Moon, and Planets, 38, 273

Krishna Swamy, K. S., Sandford, S. A., Allamandola, L. J., Witteborn, F. C., \& Bregman, J. D. 1988, Icarus, 75, 351

Levasseur-Regourd, A. C., Cabane, M., Haudebourg, V., \& Worms, J. C. 1998, Earth, Moon and Planets, 80, 343

Levasseur-Regourd, A. C., Cabane, M., Worms, J. C., et al. 1997, Adv. Space Res., 20, 1585

Li, A., \& Greengerg, M. 1997, A\&A, 323, 566

Li, A., \& Greengerg, M. 1998, ApJ, 498, L83

Licandro, J., Rubio, L. R. B., Boehnhardt, H., et al. 1998, ApJ, 501, L221-225

Manset, N. H., \& Bastien, P. 2000, Icarus, 105, 203

O'Meara, S. J., Bortle, J. E., Birkle, K., et al. 1996, 1996 August 28, ed. B. G. Marsden, IAUC, 6463

Mukai, T., Ishimoto, H., Kozasa, T., Blum, J., \& Greenberg, J. M. 1992, A\&A, 262, 315

Probstein, R. F. 1969, in Problems of Hydrodynamics and Continuum Mechanics, Soc. Industr. Appl. Math., ed. F. Bisshopp et al., 568
Rhode, J. R., \& Sinclair, A. 1992, in Explanatory Supplement to the Astronomical Almanac, Prepared by the Nautical Almanac Offices, UK \& USA, Jet Propulsion Laboratory, Bureau des Longitudes and the Time Service \& Astrometry departments, U.S. Naval Observatory, ed. P. K. Seidelmann, 325

Rouleau, F., \& Martin, P. G. 1991, ApJ, 377, 526

Scott, A., \& Duley, W. W. 1996, ApJ, 105, 401

Sekanina, Z. 1981, AJ, 86, 1741

Sekanina, Z. 1991, in Comets in the Post-Halley Era, ed. R. L. Newburn Jr., M. Neugebauer, \& J. Rahe (Kluwer, Dordrecht), 769

Sekanina, Z., \& Boehnhardt, H. 1998, Earth, Moon, and Planets, 78, 313

Sekanina, Z., \& Larson, S. M. 1984, AJ, 89, 1408

Tanga, P., Cellino, A., \& Di Martino, M. 1997, Earth, Moon, and Planets, 78, 359

Tegler, S. C., \& Romanishin, W. 2000, Nature, 407, 979

Vasundhara, R., Chakraborty, P., Hänel, A., et al. 1997, Earth, Moon, and Planets, 78, 321, Paper I

Vasundhara, R., \& Chakraborty, P. 1998, Icarus, 140, 221, Paper II

Weiss-Wrana, K., Giese, R. H., \& Zerull, R. H. 1985a, in Properties and Interactions of Interplanetary Dust, Proc. of the 85th IAU Colloq., Marseille, France, July 9-12, 1984, ed. R. H. Giese, \& P. Lamy, 219

Weiss-Wrana, K., Giese, R. H., \& Zerull, R. H. 1985b, in Properties and Interactions of Interplanetary Dust, Proc. of the 85th IAU Colloq., Marseille, France, July 9-12, 1984, ed. R. H. Giese, \& P. Lamy, 223

Wickramasinghe, N. C. 1973, in Light scattering functions for small particles, Produced and published by Adam Hilger Ltd, Ch. 5

Wolf, M. J., Clayton, G. C., \& Gibson, S. J. 1998, ApJ, 503, 815

Wooden, D. H., Harker, D. E., Woodward, C. E., et al. 1999, ApJ, 517, 1034

Worms J. C., Renard, J. B., Hadamcik, E., Levasseur-Regourd, A. C., \& Gayet, J. F. 1999, Icarus, 142, 281

Xing, Z., \& Hanner, M. S. 1997, A\&A, 324, 805 